\title{
Perioperative Strokes Following Surgical Correction of Mitral Valves: A Systematic Review and Meta-Analysis
}

\author{
Reshmi Udesh ${ }^{\text {a }}$ Piruthiviraj Natarajan ${ }^{\text {a }}$ Vinodh Jeevanantham ${ }^{c}$ \\ Thomas G. Gleason $^{\text {b Vinay Badhwar }}{ }^{d}$ Parthasarathy D. Thirumala ${ }^{a}$ \\ ${ }^{a}$ Department of Neurological Surgery, University of Pittsburgh School of Medicine, and ${ }^{b}$ Department of \\ Cardiothoracic Surgery, University of Pittsburgh Medical Center, Pittsburgh, PA, 'Saint Anthony Hospital, \\ Oklahoma City, OK, and d Division of Cardiothoracic Surgery, West Virginia University, Morgantown, WV, USA
}

\section{Keywords}

Mitral valve surgery $\cdot$ Perioperative stroke $\cdot$ In-hospital mortality $\cdot$ Morbidity

\begin{abstract}
Objective: The primary aim of this meta-analysis was to quantify the impact of perioperative strokes on stroke-related mortality after open mitral valve (MV) procedures were performed. Methods: An electronic search of the PubMed, Embase, and the Web of Science databases was performed to retrieve articles published up to December 2015, relevant to patients undergoing MV procedures. Data were extracted from the final list of 25 studies to calculate a summary OR for 30-day stroke-related mortality. Results: The stroke rate in the total sample population was $1.62 \%(73 / 4,498)$. The 30 -day all-cause mortality rate was $3.51 \%(158 / 4,498)$. The percentage of total deaths caused by stroke was $6.87 \%$. The summary OR of stroke-related mortality following MV procedures was estimated to be 7.22 (95\% Cl 4.13-12.63, $p<$ 0.0001 ). A subgroup analysis was done for studies involving concomitant MV surgery and coronary artery bypass grafting. The summary estimate of the subgroup showed an OR
\end{abstract}

\section{KARGER}

๑) 2017 S. Karger AG, Basel

E-Mail karger@karger.com

www.karger.com/ene of 8.508 (95\% Cl 1.552-46.622, $p=0.0136)$. Conclusion: Perioperative strokes following open MV procedures may be associated with more than 7 times greater odds of 30-day stroke-related mortality. They appear to be more commonly occurring than what is reported by current literature, making further studies investigating possible mechanisms and preventive measures a priority.

(c) 2017 S. Karger AG, Basel

\section{Introduction}

Perioperative strokes, a significant complication following open mitral valve (MV) procedures, have grave implications on the postoperative mortality rates and the patients' quality of life [1]. Mitral regurgitation (MR) or mitral stenosis is corrected by surgical MV repair or a mechanical/ biological MV replacement (MVR) or by percutaneous mitral clip insertion depending on the etiopathogenesis, severity of clinical symptoms, and perioperative risk. As with any open cardiac surgery, thromboembolic events play a major etiological role in adverse neurological outcomes following MV procedures [1]. 
Mechanical and bioprosthetic methods of MVR have been shown to be associated with higher rates of perioperative thromboembolic events and in-hospital mortality compared to MV repair due to the increased platelet activation and clot formation associated with prosthetic materials [1]. Most studies have small patient populations or are single-center studies; a systematic analysis of the published literature has not been performed.

Current literature reports the maximum risk of stroke to be in the immediate postoperative period ( $<30$ days) with the risk gradually declining to general population rates by the sixth postoperative month [1]. Ischemic strokes of cardiac origin have been attributed to intraoperative and postoperative thromboembolic phenomena. The most common risk factors contributing to strokes include increased thrombogenecity, concomitant atrial fibrillation, history of cerebrovascular disease, concomitant carotid artery stenosis and aortic atherosclerosis, preoperative left ventricular dysfunction cardiopulmonary bypass time exceeding $2 \mathrm{~h}$, lack of postoperative anticoagulation, and so on $[1,2]$. The incidence of perioperative strokes after coronary artery bypass grafting (CABG) has been extensively studied and reported to be around $1-5 \%$ [2]. Stroke rates following surgical correction of MVs for MR and their culpability in perioperative mortality have not been studied in detail in the same degree [1].

This work aims to systematically review the current scientific literature to evaluate the perioperative stroke rates following surgical correction of MV disease. Understanding the significant risk of debilitating neurological outcomes associated with MV procedures and their relationship with postoperative mortality in a large patient sample could help us investigate pharmacotherapeutic strategies that can help prevent thromboembolic events and lower perioperative stroke-related morbidity and mortality rates.

\section{Materials and Methods}

\section{Search Criteria and Strategy}

This systematic review was done in accordance with the Preferred Reporting Items for Systematic Reviews and Meta-Analyses (PRISMA) statement and guidelines (online suppl. PRISMA checklist; for all online suppl. material, see www.karger.com/ doi/10.1159/000477164). An electronic search of the PubMed, Embase, and the Web of Science databases was performed to retrieve a list of articles published up to December 2015, relevant to patients undergoing open MV repair or replacement. The search was done using a combination of the following terms: mitral valve repair, mitral valve replacement, mitral valve surgery, stroke, tran- sient ischemic attack, hemiparesis, hemiplegia, cerebrovascular accident, and mortality. The search history is provided in the supplement file.

\section{Study Selection}

The inclusion criteria included the following: (1) randomized clinical trials and prospective or retrospective cohort reviews; (2) studies conducted on patients undergoing MV repair/replacement; (3) studies inclusive of details on 30-day postoperative neurological outcomes and 30-day postoperative mortality data; (5) a total sample size of 50 or more patients (studies with a sample size of 50 or more were chosen to obtain an adequate sample size); (6) studies conducted on adult humans 18 years or older; (7) studies published in English, and (8) studies inclusive of an abstract.

\section{Data Extraction}

Two authors (R.U. and P.N.) independently screened all the titles and abstracts to identify and extract the studies that satisfied the inclusion criteria. Each author then subsequently created an Excel spreadsheet listing the articles to be included and excluded with a reason for elimination indicated by a number corresponding to one of the predetermined inclusion criteria, which was not fulfilled. A third author (P.D.T.) reconciled any disagreements in the inclusion/exclusion of studies following which a final list of studies was compiled. The following data were extracted from each study-author name and year, number of study patients, age and gender of study patients, number of patients who underwent isolated MV repair or replacement, number of patients who underwent combined MV repair/replacement with CABG or any other associated procedures, 30-day postoperative stroke data, and 30-day postoperative mortality data.

For this study, perioperative strokes were defined as any onset of new neurologic symptoms lasting longer than $24 \mathrm{~h}$ and occurring within 30 days of MV procedures. The main outcome of interest was 30-day mortality or in-hospital mortality rates among patients with perioperative strokes. Data on patients with perioperative strokes who died, patients with perioperative strokes not resulting in death, patients with no perioperative strokes who died, and patients with no perioperative strokes and no resultant deaths were collected for each study and tabulated in a $2 \times 2$ table, one for each category of patients.

\section{Quality Assessment of Individual Studies}

The risk of bias in the individual studies was determined based on recommendations of the Cochrane Collaboration. Twenty-four prospective and retrospective cohort studies were assessed for quality using the Newcastle-Ottawa Scale. The quality of these studies was assessed across 3 domains - selection, comparability, and outcome. According to the Newcastle-Ottawa Scale, a maximum of 4 stars may be awarded for selection, 2 stars for comparability, and 3 stars for outcome. Studies that receive 9 stars are of the highest quality. The results of the quality assessment are presented in the supplementary material (online suppl. Table 1).

\section{Statistical Analysis}

Statistical analysis was performed using version 3.1.2 of the METAFOR package for R [3]. Data extracted from each of the finalized studies were used to calculate the summary OR for 30-day stroke-related mortality outcomes in patients with perioperative 
Fig. 1. PRISMA chart exhibiting the elimi-

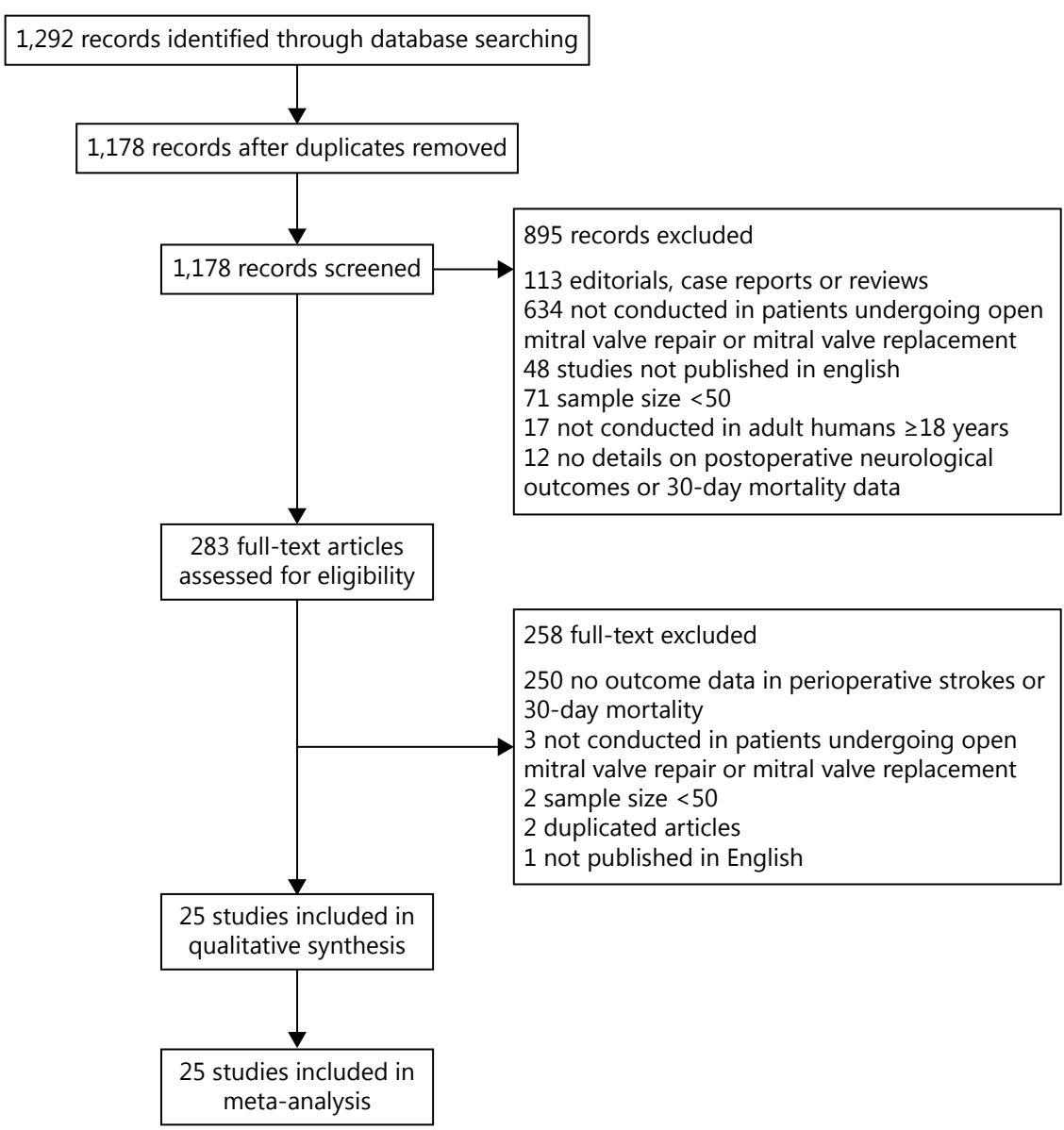
nation process for study analysis.

strokes versus patients without perioperative strokes following MV repair or replacement. A forest plot of the summary log ORs was constructed along with the calculation of the $I^{2}$ statistic to both visualize and quantify heterogeneity among the studies. For the purposes of this study, $I^{2}$ values of 25,50 , and $75 \%$ corresponded to low, medium, and high heterogeneity respectively. An overarching estimate of OR was calculated after relevant details were pooled from the individual studies using a random effects model. This type of model was used because this analysis involved a random sampling of patients undergoing MV repair or replacement. Publication bias was assessed using a funnel plot and Egger's regression test.

\section{Results}

The electronic database search revealed a total of 1,178 studies after eliminating duplicates. A preliminary screening of all titles and abstracts excluded 895 studies based on the predetermined inclusion criteria (Fig. 1). Two hundred eighty-three studies were considered for further full-text screening of which 258 studies were excluded from the final list due to the lack of sufficient data on 30-day neurological outcomes and mortality. The total study population analyzed comprised of 4,498 patients. The mean age across the patient population was 62.76 years. One study by Seeburger et al. [4], including a population of 126 patients, provided no details on patient gender. In the remaining 24 studies, $60.6 \%(2,652 / 4,372)$ were found to be male patients.

Overall, 1.62\% $(73 / 4,498)$ study patients developed perioperative strokes within 30 days of the procedure. The 30-day all-cause mortality rate was 3.51\% (158/4,498; Table 1). Of the 73 patients who developed perioperative strokes, 11 (15.1\%) died within 30 days. The percentage of patients who did not develop any 30-day strokes and survived more than 30 days following the procedure was $96.67 \%(4,278 / 4,425)$. The 30 -day mortality rate is found to be 4 times higher for patients with perioperative strokes (15.1\%) compared to patients without perioperative 


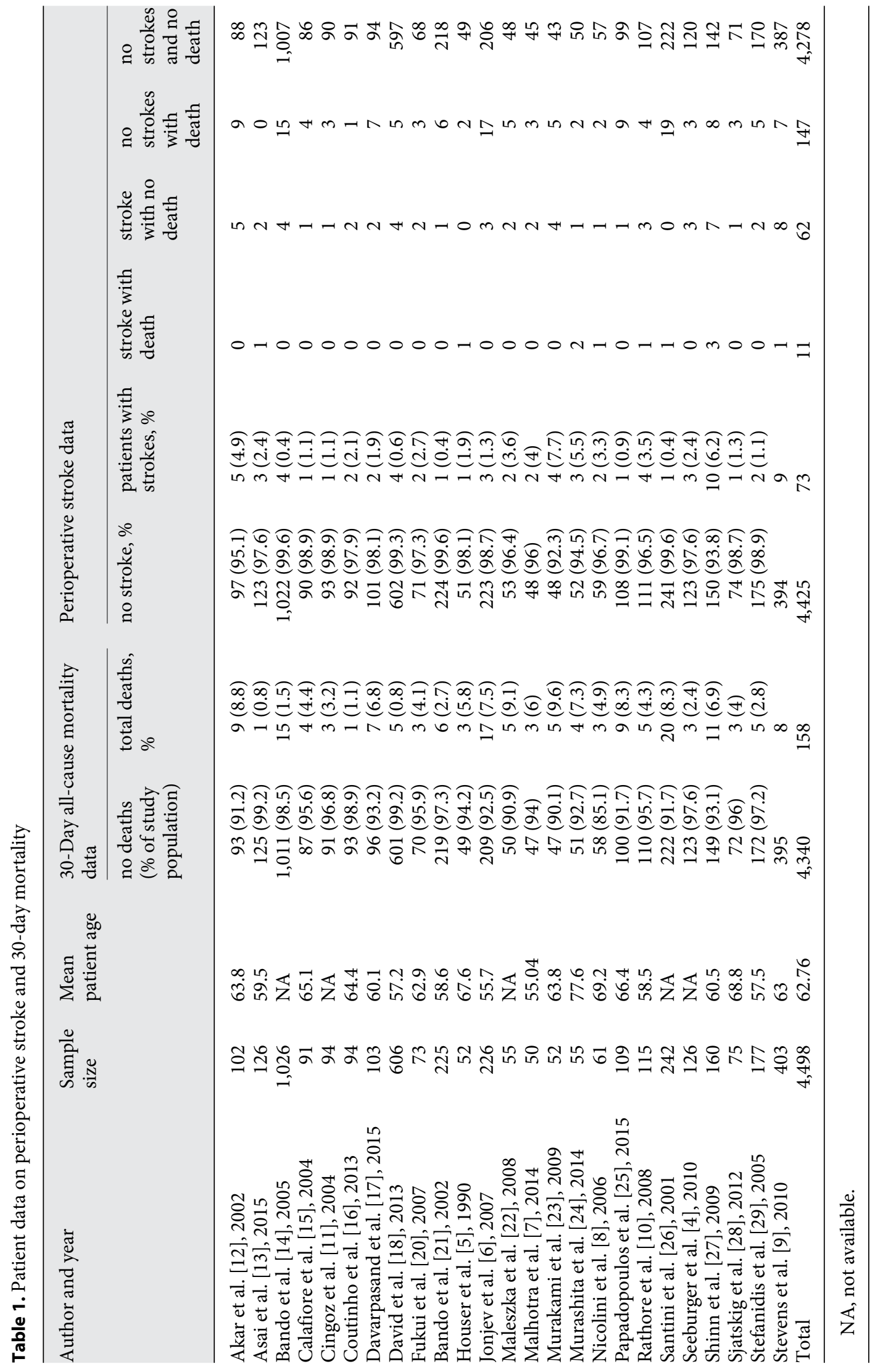


Table 2. Individual ORs

\begin{tabular}{lc}
\hline Author and year & OR (95\% CI) \\
\hline Akar et al. [12], 2002 & $0.85(0.04-16.53)$ \\
Asai et al. [13], 2015 & $148.20(4.77-4,607.42)$ \\
Bando et al. [14], 2005 & $7.22(0.37-140.0)$ \\
Calafiore et al. [15], 2004 & $6.41(0.23-180.45)$ \\
Cingoz et al. [11], 2004 & $8.62(0.29-251.65)$ \\
Coutinho et al. [16], 2013 & $12.20(0.39-379.89)$ \\
Davarpasand et al. [17], 2015 & $2.52(0.11-57.42)$ \\
David et al. [18], 2013 & $12.07(0.58-252.35)$ \\
Fukui et al. [20], 2007 & $3.91(0.16-98.04)$ \\
Bando et al. [21], 2002 & $11.21(0.42-302.09)$ \\
Houser et al. [5], 1990 & $59.40(1.89-1,859.22)$ \\
Jonjev et al. [6], 2007 & $1.69(0.08-33.97)$ \\
Maleszka et al. [22], 2008 & $1.76(0.07-41.65)$ \\
Malhotra et al. [7], 2014 & $2.60(0.10-65.41)$ \\
Murakami et al. [23], 2009 & $0.88(0.04-18.62)$ \\
Murashita et al. [24], 2014 & $50.0(3.08-810.54)$ \\
Nicolini et al. [8], 2006 & $28.50(1.27-638.92)$ \\
Papadopoulos et al. [25], 2015 & $3.49(0.13-91.78)$ \\
Rathore et al. [10], 2008 & $8.92(0.75-105.79)$ \\
Santini et al. [26], 2001 & $34.23(1.34-868.78)$ \\
Seeburger et al. [4], 2010 & $4.92(0.21-114.55)$ \\
Shinn et al. [27], 2009 & $7.61(1.65-35.08)$ \\
Sjatskig et al. [28], 2012 & $6.81(0.23-199.15)$ \\
Stefanidis et al. [29], 2005 & $6.20(0.26-145.1)$ \\
Stevens et al. [9], 2010 & $6.91(0.76-62.95)$ \\
&
\end{tabular}

strokes $(3.32 \%)$. The summary estimate across the final list of studies analyzed showed an OR of 7.22 (95\% CI $4.13-12.63, p<0.0001$ ). The ORs for the individual studies are presented in Table 2. Forest plot of the summary and individual ORs are shown in Figure 2. No asymmetry was shown in the funnel plot or Egger's regression test $(\mathrm{z}=0.1338, p=0.8936$; Fig. 3$)$.

\section{Subgroup Analysis}

A subgroup analysis was done for studies involving concomitant MV surgery (either MV repair or replacement) and CABG. Four primary studies with a sample size of 389 patients were analyzed [5-8]. A perioperative stroke rate of $2.05 \%(8 / 389)$ and 30 -day mortality rate of $6.68 \%(23 / 389)$ were obtained. The summary estimate of the risk of mortality in these 4 studies showed an OR of 8.508 (95\% CI $1.552-46.622$, $p=0.0136$ ). Only 2 studies $[9,10]$ involved isolated MV repair without any concomitant procedures. These 2 studies with a sample size of 384 patients had a perioperative stroke rate of $3.12 \%(12 / 384)$ and a 30 -day mortality rate of $0.26 \%(1 / 384)$. The calculated summary OR was $9.3686(95 \%$ CI 1.302-67.401, $p=$
0.0263). One primary study [11] involved isolated MVR with a perioperative stroke rate of $1.06 \%$. Majority of MV procedures were performed concurrently with other valvular or coronary artery procedures or atrial fibrillation surgery and this lack of adequate patients undergoing isolated MV procedures limited an extensive analysis of perioperative outcomes.

\section{Discussion}

This meta-analysis defines that surgical MV procedures have a perioperative stroke rate of $1.62 \%$ with the 30 -day mortality rate found to be 4 times higher in patients with perioperative strokes ( 15.1 vs. $3.32 \%)$. The calculated OR indicates that patients with perioperative strokes procedures have 7.22 times higher risk of 30-day stroke-related mortality compared to patients without perioperative strokes.

Studies report stroke rates ranging widely from 1 to $8 \%$ following MV surgery and when compared to the results of our study, rates are on lower end of the spectrum [2]. This discrepancy between reported stroke rates from the literature and actual clinical experience by surgeons could be explained by the incidence of subclinical silent infarcts detectable only on post-surgery MRI [30]. Although rapid advancements in anesthetic and surgical techniques have led to progressive improvements in outcomes with declining postoperative mortality rates following valve operations, postoperative ischemic neurological complications remain just as daunting as they were a decade ago [2]. A handful of studies have reported the role of subclinical infarcts following MV surgery; however, further investigation into the incidence of these lesions and their long-term implications on quality of life appears crucial [30].

In the current analysis, 2 studies $[9,10]$ reported isolated MV repair with a perioperative stroke rate of $3.12 \%$ and a summary OR of 9.36, and one study with isolated MVR [11]. The remaining studies involved one or more associated procedures like CABG, maze procedures, or aortic valve procedures depending on the patients' associated symptoms or the surgeons' judgment and restricted further subgroup analyses. Patients with ischemic MR due to coronary artery disease usually undergo MV repair or replacement concurrently with CABG. CABG has been associated with a perioperative stroke rate of $1-5 \%$ and the predisposing factors are consistent with the risk factors for perioperative stroke following MV procedures [2]. A subgroup analysis of 4 studies [5-8] with patients 
Fig. 2. Forest plot of $\log$ OR for perioperative strokes predicting mortality.

Akar, 2002

Asai, 2015

Bando, 2005

Calafiore, 2004

Cingoz, 2004

Coutinho, 2013

Davarprasand, 2015

David, 2013

Fukui, 2007

Hirata, 2002

Houser, 1990

Jonjev, 2007

Maleszka, 2008

Malhotra, 2014

Murakami, 2009

Murashita, 2014

Nicolini, 2006

Papadopoulos, 2015

Rathore, 2008

Santini, 2001

Seeburger, 2010

Shinn, 2009

Sjatskig, 2012

Stefanidis, 2005

Stevens, 2010

Tripp, 2002

RE model

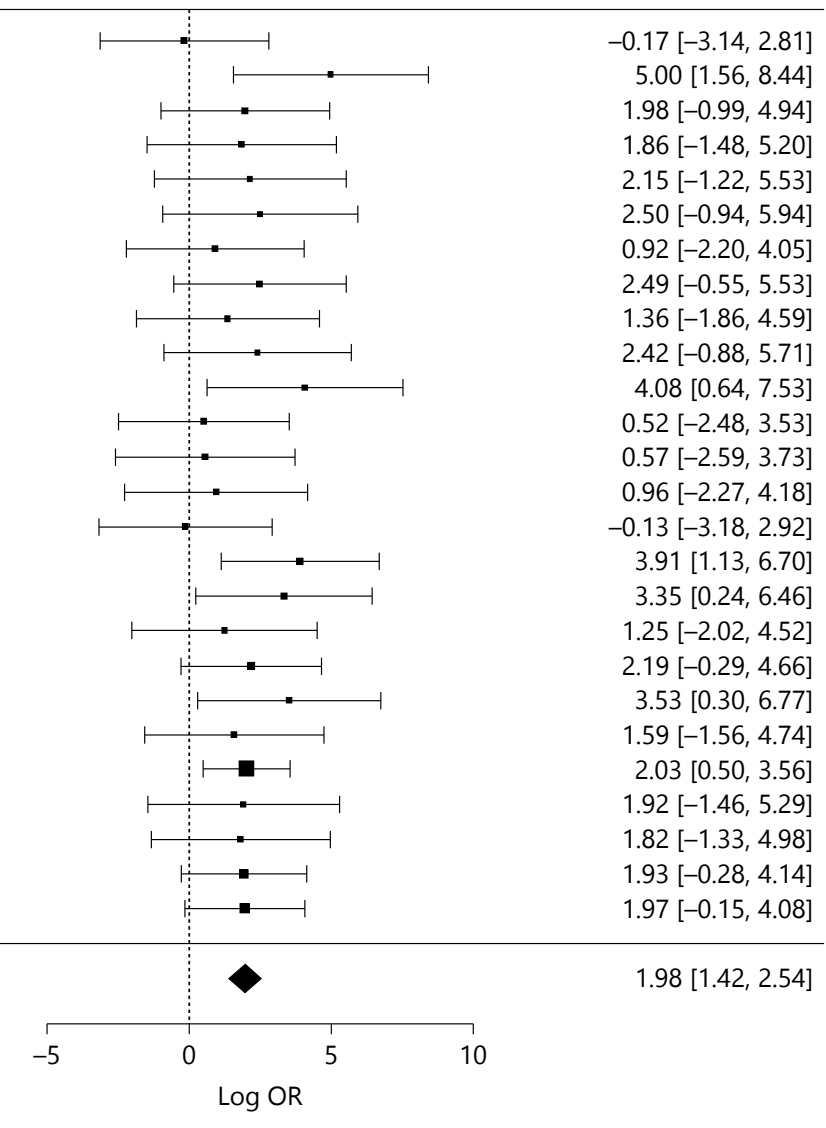

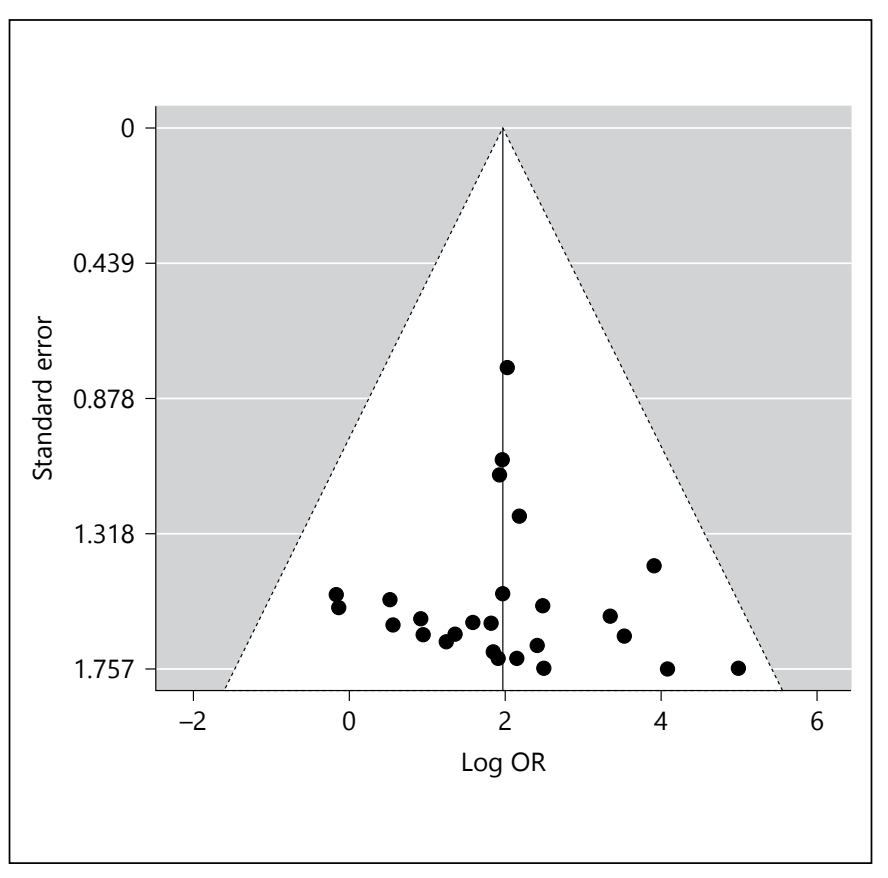

Fig. 3. Funnel Plot to detect publication bias shows no asymmetry. undergoing $\mathrm{CABG}$ with $\mathrm{MV}$ procedures had a perioperative stroke rate of $2.05 \%$ and revealed a summary OR of 8.50. Patients who undergo dual valve replacement of aortic and MV s have higher risk of perioperative thromboembolism due to greater surface area for thrombogenecity and associated left ventricular dysfunction [2]. In our study, these adjunctive procedures and their additive risks could have contributed to the perioperative stroke risk.

The significant risk of 30-day stroke-related mortality following surgical correction of regurgitant MVs warrants further comprehensive clinical studies to identify factors predisposing to perioperative strokes and thereby design-preventive pharmacotherapeutic strategies. Intraoperative neuromonitoring modalities like evoked potentials, electroencephalograms, and neuroprotection medications have shown considerable utility in predicting perioperative strokes following neurosurgical procedures like carotid endarterectomy and aneurysm clipping. These modalities may be extended to MV procedures and clinical studies to further investigate their 
applicability in preventing early stroke-related mortality may be warranted.

A few limitations are to be addressed in our study. The final list of studies analyzed gathered from different sources and locations can differ in their guidelines for MV procedures and postoperative neurological assessment and follow-up. The study, being retrospective, does not give us information on the real incidence of stroke in this population. The MV procedures were performed concurrently with other cardiac procedures based on clinical comorbidities such as coronary artery disease or atrial fibrillation and it was difficult to distinguish patients who underwent isolated MV procedures among the sample population. This may lead to variability in the final study sample. The 30-day mortality causes could not be delineated as cardiac or non-cardiac in some of the sample patients. Associated clinical findings in 30-day stroke-related deaths could not be ascertained in some analyzed studies. Other vascular risk factors such as hypertension, diabetes, and dyslipidemia and their association with perioperative stroke risk could not be measured. The protocol for postoperative neurological evaluation could have been vastly different in each hospital and could not be accounted for.

\section{Conclusion}

Perioperative stroke following surgical MV procedures may be associated with more than 7 times greater odds of 30-day stroke-related mortality. Understanding the significant risk of debilitating neurological outcomes associated with MV procedures and their relationship with early postoperative mortality may help us investigate monitoring and pharmacotherapeutic strategies, which can help prevent thromboembolic or ischemic events and lower perioperative stroke-related mortality rates.

\section{Disclosure Statement}

The authors have no conflicts of interest to disclose. There were no sources of funding for this work.

\section{References}

1 Russo A, Grigioni F, Avierinos JF, Freeman WK, Suri R, Michelena H, et al: Thromboembolic complications after surgical correction of mitral regurgitation incidence, predictors, and clinical implications. J Am Coll Cardiol 2008;51:1203-1211.

2 Bucerius J, Gummert JF, Borger MA, Walther T, Doll N, Onnasch JF, et al: Stroke after cardiac surgery: a risk factor analysis of 16,184 consecutive adult patients. Ann Thorac Surg 2003; 75:472-478.

3 Viechtbauer W: Conducting meta-analyses in $\mathrm{R}$ with the metafor package. J Stat Softw 2010; 36:1-48.

4 Seeburger J, Diegeler A, Dossche K, Lange R, Mohr FW, Schreiber C, et al: Live broadcasting in cardiac surgery does not increase the operative risk. Eur J Cardiothorac Surg 2011; 40:367-371.

5 Houser SL, Hashmi FH, Lehmann TL, Kalmbach TK, Chawla SK: Coronary artery bypass plus mitral valve replacement. A five-year study. Conn Med 1990;54:655-659.

6 Jonjev ZS, Mijatov M, Fabri M, Popovic S, Radovanovic ND: Systematic reductive annuloplasty of the mitral and tricuspid valves in patients with end-stage ischemic dilated cardiomyopathy. J Card Surg 2007;22:111-116.

7 Malhotra A, Sharma P, Garg P, Bishnoi A, Kothari J, Pujara J: Ring annuloplasty for ischemic mitral regurgitation: a single center experience. Asian Cardiovasc Thorac Ann 2014; 22:781-786.
8 Nicolini F, Zoffoli G, Cagnoni G, Agostinelli A, Colli A, Fragnito C, et al: Mitral valve annuloplasty and myocardial revascularization in the treatment of ischemic dilated cardiomyopathy. Heart Vessels 2006;21:2832.

9 Stevens LM, Basmadjian AJ, Bouchard D, ElHamamsy I, Demers P, Carrier M, et al: Late echocardiographic and clinical outcomes after mitral valve repair for degenerative disease. J Card Surg 2010;25:9-15.

10 Rathore KS, Kumar P, Jadhav U, Tendolkar AG: Rheumatic mitral valve surgery in the fifth decade: our experience. J Cardiovasc Surg (Torino) 2008;49:119-124

11 Cingoz F, Gunay C, Kuralay E, Yildirim V, Kilic S, Demirkilic U, et al: Both leaflet preservation during mitral valve replacement: modified anterior leaflet preservation technique. J Card Surg 2004;19:528-534.

12 Akar R, Doukas G, Szafranek A, Alexiou C, Boehm MC, Chin D, et al: Mitral valve relpair and revascularization for ischemic mitral regurgitation: predictors of operative mortality and survival. J Heart Valve Dis 2002;11:793800; discussion 801.

13 Asai T, Kinoshita T, Suzuki T, Shiraishi S, Koike M: Early and follow-up results of butterfly resection of prolapsed posterior leaflet in 76 consecutive patients. J Thorac Cardiovasc Surg 2015;149:1296-1300.

14 Bando K, Kasegawa H, Okada Y, Kobayashi J, Kada A, Shimokawa T, et al: Impact of pre- operative and postoperative atrial fibrillation on outcome after mitral valvuloplasty for nonischemic mitral regurgitation. J Thorac Cardiovasc Surg 2005;129:10321040.

15 Calafiore M, Di Mauro M, Gallina S, Yassin IM, Shaheen YA, Canosa C, et al: Surgical treatment of mitral valve regurgitation in dilated cardiomyopathy. Heart Surg Forum 2004;7:E67-E71.

16 Coutinho GF, Correia PM, Pancas R, Antunes MJ: Management of moderate secondary mitral regurgitation at the time of aortic valve surgery. Eur J Cardiothorac Surg 2013;44:3240.

17 Davarpasand T, Hosseinsabet A, Jalali A: Concomitant coronary artery bypass graft and aortic and mitral valve replacement for rheumatic heart disease: short- and mid-term outcomes. Interact Cardiovasc Thorac Surg 2015;21:322-328.

18 David TE, Armstrong S, Ivanov J: Chordal replacement with polytetrafluoroethylene sutures for mitral valve repair: a 25-year experience. J Thorac Cardiovasc Surg 2013;145: 1563-1569.

19 David TE, Omran A, Armstrong S, Sun Z, Ivanov J: Long-term results of mitral valve repair for myxomatous disease with and without chordal replacement with expanded polytetrafluoroethylene sutures. J Thorac Cardiovasc Surg 1998;115:1279-1285; discussion $1285-1286$. 
20 Fukui T, Shibata T, Sasaki Y, Hirai H, Motoki $\mathrm{M}$, Takahashi $\mathrm{Y}$, et al: Long-term experience with the Sorin Bicarbon and Edwards Mira mechanical valve prostheses in the mitral position. Gen Thorac Cardiovasc Surg 2007;55: 91-97.

21 Bando K, Kobayashi J, Sasako Y, Tagusari O, Niwaya K, et al: Effect of maze procedure in patients with atrial fibrillation undergoing valve replacement. J Heart Valve Dis 2002;11: 719-724; discussion 725.

22 Maleszka A, Kleikamp G, Zittermann A, Serrano MR, Koerfer R: Simultaneous aortic and mitral valve replacement in octogenarians: a viable option? Ann Thorac Surg 2008; 86: 1804-1808.

23 Murakami M, Yamaguchi H, Suda Y, Asai T, Sueishi M, Matsumura T: Mitral valve repair for 52 patients with severe left ventricular dysfunction. Ann Thorac Cardiovasc Surg 2009; 15:160-164.
24 Murashita T, Okada Y, Kanemitsu H, Fukunaga N, Konishi $\mathrm{Y}$, Nakamura K, et al: Feasibility and efficacy of mitral valve repair for degenerative mitral regurgitation in the elderly. Ann Thorac Cardiovasc Surg 2014;20: 1001-1007.

25 Papadopoulos N, Steuer K, Doss M, Moritz A, Zierer A: Is removal of calcium bar during mitral valve surgery safe? Long-term clinical outcome of 109 consecutive patients. J Cardiovasc Surg (Torino) 2015;56:473-482.

26 Santini F, Auriemma S, Casali G, Rossi A, Lusini M, Mazzucco A: Long-term results with the xenomedica porcine bioprosthesis in the mitral position. J Heart Valve Dis 2001; 10: 789-794.
27 Shinn $\mathrm{SH}$, Oh SS, Na CY, Lee $\mathrm{CH}$, Lim $\mathrm{HG}$, Kim JH, et al: Short- and longterm results of triple valve surgery: a single center experience. J Korean Med Sci 2009;24:818-823.

28 Sjatskig J, Yilmaz A, van Boven JW, Sonker U, Waanders FG, Kloppenburg GT: Feasibility of mitral valve surgery using minimal extracorporeal circulation. Perfusion 2012;27: 264-268.

29 Stefanidis C, Nana AM, De Canniere D, Antoine M, Jansens JL, Huynh CH, et al: 10-Year experience with the ATS mechanical valve in the mitral position. Ann Thorac Surg 2005;79: 1934-1938.

30 Floyd TF, Shah PN, Price CC, Harris F, Ratcliffe SJ, Acker MA, et al: Clinically silent cerebral ischemic events after cardiac surgery: their incidence, regional vascular occurrence, and procedural dependence. Ann Thorac Surg 2006;81:2160-2166. 